University of Nebraska - Lincoln

DigitalCommons@University of Nebraska - Lincoln

\title{
Effect of Phosphorus Nutrition on Growth and Physiology of Cotton Under Ambient and Elevated Carbon Dioxide
}

\author{
S. K. Singh \\ G. B. Badgujar \\ USDA-ARS Beltsville \\ V. R. Reddy \\ USDA-ARS Beltsville \\ D. H. Fleisher \\ USDA-ARS Beltsville, david.fleisher@ars.usda.gov \\ D. J. Timlin \\ USDA-ARS Beltsville
}

University of Maryland Queenstown, shardendu.singh@ars.usda.gov

Follow this and additional works at: https://digitalcommons.unl.edu/usdaarsfacpub

Singh, S. K.; Badgujar, G. B.; Reddy, V. R.; Fleisher, D. H.; and Timlin, D. J., "Effect of Phosphorus Nutrition on Growth and Physiology of Cotton Under Ambient and Elevated Carbon Dioxide" (2013). Publications from USDA-ARS / UNL Faculty. 1298.

https://digitalcommons.unl.edu/usdaarsfacpub/1298

This Article is brought to you for free and open access by the U.S. Department of Agriculture: Agricultural Research Service, Lincoln, Nebraska at DigitalCommons@University of Nebraska - Lincoln. It has been accepted for inclusion in Publications from USDA-ARS / UNL Faculty by an authorized administrator of DigitalCommons@University of Nebraska - Lincoln. 
MINERAL DEFICIENCY STRESS

\title{
Effect of Phosphorus Nutrition on Growth and Physiology of Cotton Under Ambient and Elevated Carbon Dioxide
}

\author{
S. K. Singh ${ }^{1,2}$, G. B. Badgujar ${ }^{2,3}$, V. R. Reddy ${ }^{2}$, D. H. Fleisher ${ }^{2}$ \& D. J. Timlin ${ }^{2}$ \\ 1 Wye Research and Education Center, University of Maryland Queenstown, MD, USA \\ 2 Crop Systems and Global Change Laboratory, USDA-ARS Beltsville, MD, USA \\ 3 Asian Institute of Technology Pathumthani, Thailand
}

\author{
Keywords \\ biomass partitioning; nitrogen; \\ photosynthesis; specific leaf weight; stomatal \\ conductance \\ Correspondence \\ S. K. Singh \\ Crop Systems and Global Change Laboratory \\ United States Department of Agriculture \\ Agricultural Research Service \\ Bldg 001, Rm 342, BARC-West \\ 10300 Baltimore Ave. \\ Beltsville \\ MD 20705, USA \\ Tel.: +13015046633 \\ Fax: +13015045823 \\ Email: shardendu.singh@ars.usda.gov or \\ singh.shardendu@gmail.com
}

Accepted June 19, 2013

doi:10.1111/jac. 12033

\begin{abstract}
Phosphorous deficiency in soil limits crop growth and productivity in the majority of arable lands worldwide and may moderate the growth enhancement effect of rising atmospheric carbon dioxide $\left(\mathrm{CO}_{2}\right)$ concentration. To evaluate the interactive effect of these two factors on cotton (Gossypium hirsutum) growth and physiology, plants were grown in controlled environment growth chambers with three levels of phosphate (Pi) supply $(0.20,0.05$ and $0.01 \mathrm{~mm})$ under ambient and elevated (400 and $800 \mu \mathrm{mol} \mathrm{mol}{ }^{-1}$, respectively) $\mathrm{CO}_{2}$. Phosphate stress caused stunted growth and resulted in early leaf senescence with severely decreased leaf area and photosynthesis. Phosphate stress led to over $77 \%$ reduction in total biomass across $\mathrm{CO}_{2}$ levels. There was a below-ground (roots) shift in biomass partitioning under Pi deficiency. While tissue phosphorus $(\mathrm{P})$ decreased, tissue nitrogen $(\mathrm{N})$ content tended to increase under $\mathrm{Pi}$ deficiency. The $\mathrm{CO}_{2} \times \mathrm{Pi}$ interactions were significant on leaf area, photosynthesis and biomass accumulation. The stimulatory effect of elevated $\mathrm{CO}_{2}$ on growth and photosynthesis was reduced or highly depressed suggesting an increased sensitivity of cotton to $\mathrm{Pi}$ deficiency under elevated $\mathrm{CO}_{2}$. Although, tissue $\mathrm{P}$ and stomatal conductance were lower at elevated $\mathrm{CO}_{2}$, these did not appear to be the main causes of cotton unresponsiveness to elevated $\mathrm{CO}_{2}$ under severe Pi-stress. The alteration in the uptake and utilization of $\mathrm{N}$ was suggested due to a consistent reduction $(18-21 \%)$ in the cotton plant tissue $\mathrm{N}$ content under elevated $\mathrm{CO}_{2}$.
\end{abstract}

\section{Introduction}

Phosphate $(\mathrm{Pi})$ deficiency in soil is a limiting growth factor in over $30 \%$ of crop lands, and a major production constraint in acidic soils comprising up to $70 \%$ of arable lands worldwide (Vance et al. 2003, Cordell et al. 2009, Lenka and Lal 2012). Phosphate is a component of nucleic acids and cellular membranes, and essential for metabolic processes (Raghothama 1999, Vance et al. 2003). Phosphate deficiency decreases plant growth and photosynthesis and thus biomass accumulation and yield. The crop demand for nutrients such as $\mathrm{Pi}$ has increased due to the introduction of high yielding cultivars, and this requirement may be even higher due to increased plant growth under rising atmospheric carbon dioxide concentrations $\left(\mathrm{CO}_{2}\right)$ (Rogers et al. 1993, Lewis et al. 1994, Lenka and Lal 2012). The cur- rent atmospheric $\mathrm{CO}_{2}$ concentration of approximately $394 \mu \mathrm{mol} \mathrm{mol}^{-1}$ is projected to be doubled by the end of 21st century (IPCC 2007). In general, elevated $\mathrm{CO}_{2}$ enhances plant growth, photosynthesis and yield. However, soil nutrients such as $\mathrm{Pi}$ exert major control over plant response to elevated $\mathrm{CO}_{2}$ (Lewis et al. 1994, Campbell and Sage 2006, Lenka and Lal 2012). Studies evaluating the effect of Pi deficiency on cotton growth and physiological processes under current and projected atmospheric $\mathrm{CO}_{2}$ conditions are limited. Because phosphate deficiency and elevated $\mathrm{CO}_{2}$ may co-exist under natural environments, which have an opposite effect on plant growth and productivity, it is imperative to evaluate the crop responses to the interaction between these two factors.

Cotton (Gossypium hirsutum) is an important fibre crop grown worldwide (FAO 2010). Cotton growth, 
photosynthesis and lint yield respond positively to elevated $\mathrm{CO}_{2}$ and availability of nutrients including Pi (Sawan 1986, Kimball and Mauney 1993, Rogers et al. 1993, Singh et al. 2006, Girma et al. 2007). However, the positive response of elevated $\mathrm{CO}_{2}$ is often reduced when cotton is grown in combination with low supply of nutrients such as nitrogen (Rogers et al. 1993), potassium (Reddy and Zhao 2005) and phosphorus (Rogers et al. 1993, Barrett and Gifford 1995). Nutrient deprivation caused imbalance in hydraulic conductance, decreased cell expansion and decreased biosynthesis of photosynthetic pigments and metabolic enzymes, which may inhibit the stimulatory effect of elevated $\mathrm{CO}_{2}$ on plant growth (Radin and Eidenbock 1984, Rogers and Humphries 2000). Under field conditions, crops are routinely exposed to multiple stress factors; therefore, understanding of the interaction between environmental factors such as $\mathrm{CO}_{2}$ and $\mathrm{Pi}$ nutrition is critical for nutrient management.

The $\mathrm{CO}_{2}$ enrichment and deficiency of nutrients including Pi have also been reported to affect biomass partitioning and nutrient allocation to plant organs (leaves, stems and roots) (Prior et al. 1998, Reddy and Zhao 2005, Fleisher et al. 2012). Moreover, the composition of other nutrients such as nitrogen $(\mathrm{N})$ in plant tissues may be altered due to changes in nutrient uptake and utilization under $\mathrm{Pi}$-stress as well as elevated $\mathrm{CO}_{2}$ leading to more complex interactions between these two factors (Prior et al. 1998, Fleisher et al. 2012, 2013, Lenka and Lal 2012). Therefore, nutrient availability will play a vital role in determining the magnitude and direction of plant growth and physiological response to $\mathrm{CO}_{2}$ enriched environment.

Phosphorus deficiency inhibits cotton growth and development by decreasing photosynthetic capacity (Longstreth and Nobel 1980, Radin and Eidenbock 1986, Barrett and Gifford 1995), leaf expansion (Radin and Eidenbock 1984), biomass accumulation (Radin and Eidenbock 1984, Ahmad et al. 2001) and yield (Singh et al. 2006). Rogers et al. (1993) suggested that the critical concentration of $\mathrm{Pi}$ required for maximum crop productivity may increase in a $\mathrm{CO}_{2}$ enriched environment. Thus, plants currently not limited by Pi may become limited due to projected increases in atmospheric $\mathrm{CO}_{2}$ (Lewis et al. 1994, Lenka and Lal 2012). Studies evaluating cotton growth and physiology under varying levels of Pi supply and $\mathrm{CO}_{2}$ are limited (Gifford et al. 2000). Most of the prior studies investigating crop responses under varying nutrient supply have focused on nutrients other than $\mathrm{Pi}$ and few have considered major row crops including cotton and their interaction with $\mathrm{CO}_{2}$ concentrations (Jacob and Lawlor 1991, Reddy and Zhao 2005, Campbell and Sage 2006, Bown et al. 2009, Jin et al. 2011). We hypothesize that severe Pi deficiency may limit/ offset the positive effects of elevated $\mathrm{CO}_{2}$ on cotton growth and physiological processes due to increased sensitivity.
The objectives of this study were to determine the interactive effects of $\mathrm{CO}_{2}$ and $\mathrm{Pi}$ supply on cotton growth and physiological processes and quantify the allocation of biomass and nutrients in plant organs.

\section{Materials and Methods}

\section{Growth conditions}

The study consisted of two experiments in 2011, hereafter referred to as Exp. I and Exp. II, which were conducted at USDA-ARS Henry A. Wallace Agricultural Research Center in Beltsville, MD USA. Both experiments used the same six controlled environment growth chambers (EGC Corp., Chagrin Falls, OH, USA) but at different dates and were completed in 112 (Exp. I) and 91 days (Exp. II) from the day after planting (DAP). In addition, these experiments differed from one another with respect to the number and size of pots used, initiation of $\mathrm{CO}_{2}$ treatments and frequency and timing of the measurements.

In Exp. I, cotton (cv. Deltapine 555) seeds were planted in 12 pots (volume, 16-1) filled with fine sand and vermiculite (3: 1 volume ratio) in each of the six growth chambers. Two levels (400 and $800 \mu \mathrm{mol} \mathrm{mol}^{-1}$ ) of $\mathrm{CO}_{2}$ treatments were initiated each in three chambers from the beginning, and plants were watered with full strength Hoagland's nutrient solution (Hewitt 1952) from the emergence to 34 days after planting (DAP). Thereafter, phosphate treatments (for simplicity referred as $\mathrm{Pi}$ ) were initiated with the modified Hoagland's nutrient solution by three levels of Pi treatments, 0.20, 0.05 and $0.01 \mathrm{~mm}$ each at two levels of $\mathrm{CO}_{2}$. The Pi treatments were selected based on the previous studies with Pi nutrition in cotton reflecting a range in Pi-stress from $0 \%$ up to $33 \%$ compared with the full nutrient supply (Radin and Eidenbock 1984, 1986, Barrett and Gifford 1995). The selected treatments correspond to the full nutrient $(0.20 \mathrm{~mm})$ and Pi-stresses of $25 \%(0.05 \mathrm{~mm})$ and $5 \%(0.01 \mathrm{~mm})$ of the full. A range in the tissue $\mathrm{P}$ content was obtained using the current $\mathrm{Pi}$ treatments.

In Exp. II, cotton seeds from the same cultivar were planted outdoor in 120 pots on 27 July 2011 (volume 7.61), containing rooting medium similar to the Exp. I. Plants were watered with full strength Hoagland's nutrient solution from emergence to 34 DAP. Thereafter, plants were transferred and randomly assigned to the six growth chambers (20 pots each), and the treatments (two levels of $\mathrm{CO}_{2}$ and three levels of Pi) were initiated same as in Exp. I. At this stage, there were no statistical differences between chambers $(P>0.975)$ for plant height, leaf area and number of leaves plant $^{-1}$.

For both experiments, the daytime temperature and light regime were initiated at 6:00 h. The light as 
photosynthetically active radiation (PAR) of $800 \mu \mathrm{mol}$ $\mathrm{m}^{-2} \mathrm{~s}^{-1}$ at plant canopy was supplied during day period using a combination of metal halide and high pressure sodium lamps. A $30 / 22{ }^{\circ} \mathrm{C}$ day/night (14-h day and 10 -h night) temperature was maintained in the growth chambers during experiments. Injection of either $\mathrm{CO}_{2}$ - or $\mathrm{CO}_{2}$-free air was determined using a TC-2 controller that monitored $\mathrm{CO}_{2}$ measured from an absolute infrared gas analyser (WMA-2 PP-systems, Haverhill, MA, USA). Relative humidity varied between 50 and $70 \%$ during the experiment among the chambers.

\section{Growth measurements}

For each experiment, plant height $(\mathrm{PH})$ and leaf length on each main stem nodes were measured weekly in six (Exp. I) and eight plants (Exp. II) from 50 (Exp. I) and 34 (Exp. II) DAP to the end of experiments. The leaf lengths were subsequently converted to main stem leaf area (MSLA) using a quadratic equation $y=0.9569 x^{2}-2.0162 x+0.8811$, $\left(\mathrm{r}^{2}=0.96, \mathrm{n}=253, y=\right.$ area in $\mathrm{cm}^{2}$ and $x=$ leaf length in $\mathrm{cm}$ ) established by developing a relationship between the lengths of different leaves and leaf area, measured using a LICOR-3100 leaf area metre (LICOR, Inc., Lincoln, NE, USA) at the time of destructive harvests. Plants were harvested at 85 and 112 DAP (six pots each) in Exp. I, and 67, 81 and 93 DAP (six, six and eight pots, respectively) in Exp. II. Plants were separated into stems, leaves, fruits (includes flowers, squares and bolls) and roots, and plant height (PH), number of main stem nodes (MSN), total leaf area (TLA) and total biomass (TBM) were measured. Roots were washed in clean water. The dry weight of plant materials were determined after drying in an oven at $70{ }^{\circ} \mathrm{C}$ until constant weight was obtained.

\section{Gas exchange measurements}

In both experiments, leaf photosynthetic rate $\left(P_{\text {net }}\right)$ and stomatal conductance $\left(g_{\mathrm{s}}\right)$ were measured on the upper most fully expanded leaves between 9:00 and 13:00 h using a portable LICOR 6400 Photosynthesis system (LICOR Inc.). This photosynthesis system used blue $(475 \mathrm{~nm})$ and red light-emitting diodes (LED) $(630 \mathrm{~nm})$ as a light source mounted onto a two $\mathrm{cm}^{2}$ leaf chamber. The gas exchange measurements were recorded when a steady-state (around 3-6 min) was obtained, at $1500 \mu \mathrm{mol}$ photon $\mathrm{m}^{-2} \mathrm{~s}^{-1}$ photosynthetically active radiation (PAR). The leaf cuvette temperature was set to $30{ }^{\circ} \mathrm{C}$. The $\mathrm{CO}_{2}$ entering in the cuvette was maintained at the treatment $\mathrm{CO}_{2}$ concentration level of either 400 or $800 \mu \mathrm{mol} \mathrm{mol}^{-1}$ and relative humidity varied between 45 and $60 \%$. The photosynthetic rate, $g_{s}$, and intercellular $\left(\mathrm{C}_{\mathrm{i}}\right)$ to external $\left(\mathrm{C}_{\mathrm{a}}\right) \mathrm{CO}_{2}$ concentration ratio $\left(\mathrm{C}_{\mathrm{i}} / \mathrm{C}_{\mathrm{a}}\right)$ were automatically computed from instru- ments software (details are available in LI-6400 Instruction Manual, version 5, Li-Cor Inc., Lincoln, Nebraska, USA).

\section{Measurement of tissue constituents}

Total chlorophyll concentration, tissue phosphorus (for simplicity and to distinguish from phosphate treatments, referred as $\mathrm{P}$ ), carbon $(\mathrm{C})$ and nitrogen $(\mathrm{N})$ contents were determined from the upper most fully expanded leaves that were used for gas exchange measurements, and in plant organs (leaves, stems and roots) after each harvest. The individual leaf area and dry weight were also recorded for uppermost fully expanded leaves to determine specific leaf weight (SLW, mg dry weight $\mathrm{cm}^{-2}$ leaf area). The tissue $\mathrm{P}$, $\mathrm{C}$ and $\mathrm{N}$ contents in uppermost fully expanded leaves were expressed either based on leaf dry mass (mass-based, mg $\mathrm{g}^{-1}$ ) or leaf area (area-based, $\mathrm{mg} \mathrm{cm}^{-2}$ ). Whole plant tissue $\mathrm{P}$ and $\mathrm{N}$ contents were estimated as the product of dry mass of plant organs (leaves, stems and roots) and their nutrient concentration $\left(\mathrm{mg} \mathrm{g}^{-1}\right)$. Chlorophyll was extracted by placing two $0.95 \mathrm{~cm}^{2}$ leaf discs for each leaf in a vial containing $5 \mathrm{ml}$ of dimethyl sulfoxide and incubating in the dark for $24 \mathrm{~h}$. Thereafter, the absorbance of the supernatant was measured at 664 and $648 \mathrm{~nm}$ using a UV2101-PC spectrophotometer (Shimadzu Corp., Columbia, MD, USA). The total chlorophyll was estimated using the equation of Lichtenthaler (1987) and expressed on leaf area basis $\left(\mu \mathrm{g} \mathrm{cm}^{-2}\right)$. The dry tissues were ground using a Wiley Mill (Wiley ${ }^{\circledR}$ Mill, Thomas Scientific, NJ, USA) to pass through 1-mm screen. The tissue P content was quantified in the Soil Testing and Plant Analysis Laboratory, Extension Service, Mississippi State University, MS. The tissue carbon and $\mathrm{N}$ contents were determined by combustion using a CHN-2000 (Carbon Hydrogen Nitrogen-2000: LECO Corporation, St. Joseph, MI, USA).

\section{Data analysis}

All statistical analyses were performed using SAS procedures (SAS Enterprise Guide, 4.2, SAS Institute Inc., NC, USA). Proc Mixed procedure of SAS was used for regression analysis, to calculate the coefficients and to test for common slope and intercepts. Days after planting were treated as the continuous variable, and $\mathrm{CO}_{2}$ and $\mathrm{Pi}$ treatments as qualitative factors. Proc Mixed was also used to test the treatment differences among the measured variables using individual pots as pseudoreplicates between two levels of $\mathrm{CO}_{2}$ and three levels of $\mathrm{Pi}$, and chamber as a random effect. The treatment comparisons were conducted by least square means (LSMEANS) procedure with letter grouping obtained using the pdmix800 macro (Saxton 1998). In both experiments, the data were analysed separately for each harvest. Because there were no significant 
differences for leaf level measurements between the two experiments, the data measured on uppermost fully expanded leaves were combined.

\section{Results}

\section{Plant growth}

In both the experiments regardless of the harvest dates, low Pi treatments $(0.05$ and $0.01 \mathrm{~mm})$ significantly $(\mathrm{P}<0.05)$ decreased almost all the growth parameters, whereas elevated $\mathrm{CO}_{2}$ caused significant $(\mathrm{P}<0.05)$ increases in growth, mostly in high Pi-treated plants (Table 1). Plant height, MSN and MSLA followed asymptotically linear growth patterns during crop development, which were more obvious in the plants grown at $0.20 \mathrm{~mm}$ Pi (Fig. 1a-f). However, under Pi-stress treatments, MSLA started to decline 75 DAP in both experiments due to leaf senescence (Fig. 1b,e). At the final harvest, compared with the $0.20 \mathrm{~mm}$ Pi treatment, Pi-stress treatments (averaged 0.05 and $0.01 \mathrm{~mm}$ ) caused approximately 52, 25 and $85 \%$ decline in PH, MSN and TLA, respectively, across experiments, whereas TBM decreased by $89.4 \%$ (Exp. I) and
$77.7 \%$ (Exp. II). Averaged across Pi treatments, cotton grown at elevated $\mathrm{CO}_{2}$ had significantly $(\mathrm{P}<0.05)$ taller plants (18.5-23.0\%), higher TLA (10-28\%) and TBM $(31-33 \%)$ at the final harvest across experiments (Table 1). The $\mathrm{CO}_{2}$ treatment did not affect MSN of cotton plants. The averaged specific leaf weight (calculated using all leaves) tended to be slightly higher under Pi-stress and at elevated $\mathrm{CO}_{2}$.

\section{Biomass partitioning}

At final harvest in both experiments, Pi treatment had significant $(\mathrm{P}<0.001)$ effect on dry matter partitioning between plant organs (leaves, stems, fruits and roots), and when averaged across $\mathrm{CO}_{2}$ levels, Pi-stress treatments (averaged 0.05 and $0.01 \mathrm{~mm} \mathrm{Pi}$ ) exhibited $>80 \%$ decreases in biomass accumulation in each of the components as compared with $0.20 \mathrm{~mm} \mathrm{Pi}$ treatment (Table 2). The $\mathrm{CO}_{2} \times \mathrm{Pi}$ interactions were significant $(\mathrm{P}<0.01)$ for leaves and stems in both experiments. Averaged across Pi treatments, elevated $\mathrm{CO}_{2}$ significantly $(\mathrm{P}<0.01)$ increased leaf and stem biomass; however, this increment was not significant $(\mathrm{P}>0.05)$ for fruit and root biomass. The

Table 1 Effect of $\mathrm{CO}_{2}$ levels $\left(\mu \mathrm{mol} \mathrm{mol}^{-1}\right.$ ) and phosphate ( $\left.\mathrm{Pi}, \mathrm{mm}\right)$ supply on plant height $(\mathrm{PH}, \mathrm{cm})$, number of main stem nodes (MSN plant $\left.{ }^{-1}\right)$, total leaf area (TLA, $\mathrm{cm}^{2}$ plant $^{-1}$ ), total biomass (TBM, g plant ${ }^{-1}$ ) and specific leaf weight (SLW, $\mathrm{mg} \mathrm{cm}^{-2}$ of whole plant leaves) of cotton, in Exp. I (84 and 112 days after planting, DAP) and Exp. II (67 and 91 DAP). Analysis of variance (ANOvA) between Pi and $\mathrm{CO}_{2}$ is given. The data are the mean of six-eight individual plants

\begin{tabular}{|c|c|c|c|c|c|c|c|c|c|c|c|}
\hline $\mathrm{CO}_{2}$ & $\mathrm{Pi}$ & $\mathrm{PH}$ & MSN & TLA & TBM & SLW & $\mathrm{PH}$ & MSN & TLA & TBM & SLW \\
\hline \multicolumn{12}{|c|}{ Experiment I } \\
\hline & & & & 84 DAP & & & & & $112 \mathrm{DA}$ & & \\
\hline \multirow[t]{3}{*}{400} & 0.20 & $47.51 b$ & $21.5 b$ & $1993 b$ & $61.98 b$ & $10.71 b$ & $57.67 b$ & $27 a$ & $3177 b$ & $115.66 b$ & $9.40 c$ \\
\hline & 0.05 & $23.43 d$ & $18.0 \mathrm{~cd}$ & $368 c d$ & $10.02 d$ & $11.33 b$ & $31.17 d$ & $21 b c$ & $985 c$ & $29.38 c$ & $12.15 a b$ \\
\hline & 0.01 & 20.00de & 16.6de & $244 c d$ & $8.38 d$ & $12.14 \mathrm{ab}$ & $23.42 \mathrm{e}$ & $21 b c$ & $92 \mathrm{e}$ & $5.63 d$ & $9.23 c$ \\
\hline \multirow[t]{3}{*}{800} & 0.20 & $64.66 a$ & $24.6 a$ & $3714 a$ & $88.08 a$ & $10.75 b$ & $73.00 a$ & $29 a$ & $4112 a$ & $173.42 a$ & 11.71abc \\
\hline & 0.05 & $36.00 c$ & $19.0 \mathrm{bc}$ & $503 c$ & $21.94 c$ & $13.50 a$ & $39.28 c$ & $23 b$ & $516 d$ & $22.95 c$ & $12.25 a$ \\
\hline & 0.01 & $24.00 \mathrm{e}$ & $16.0 \mathrm{e}$ & $89 d$ & $7.63 d$ & $12.40 \mathrm{ab}$ & $25.70 \mathrm{e}$ & $19 c$ & $67 e$ & $3.34 d$ & $9.55 b c$ \\
\hline \multirow[t]{4}{*}{ ANOVA } & $\mathrm{CO}_{2}$ & $* * *$ & ns & $* * *$ & $* * *$ & ns & $* * *$ & ns & $*$ & $* * *$ & ns \\
\hline & $\mathrm{Pi}$ & $* * *$ & $* * *$ & $* * *$ & $* * *$ & $*$ & $* * *$ & $* * *$ & $* * *$ & $* * *$ & $*$ \\
\hline & $\mathrm{CO}_{2} \times \mathrm{Pi}$ & $* * *$ & $*$ & $* * *$ & $* * *$ & ns & $* * *$ & ns & $* * *$ & $* * *$ & ns \\
\hline & & \multicolumn{5}{|c|}{$67 \mathrm{DAP}$} & \multicolumn{5}{|c|}{91 DAP } \\
\hline \multirow[t]{3}{*}{400} & 0.20 & $35.40 \mathrm{a}$ & $15.6 a$ & $971 b$ & $16.49 b$ & $6.64 c$ & $60.65 b$ & $21 a$ & $2286 b$ & $62.45 b$ & $7.82 d$ \\
\hline & 0.05 & $25.13 b c$ & $14.1 b$ & $400 c$ & $10.12 c$ & 9.39ab & $30.84 d$ & $16 b$ & $444 c$ & $15.41 c$ & $9.20 \mathrm{~cd}$ \\
\hline & 0.01 & $21.90 c$ & $12.4 \mathrm{C}$ & $274 c$ & $7.86 c$ & $9.67 a b$ & $28.59 d$ & $16 b$ & $435 c$ & $14.73 c$ & $9.67 b c$ \\
\hline \multirow[t]{3}{*}{800} & 0.20 & $39.42 a$ & $16.3 a$ & $1208 a$ & $24.21 a$ & $8.46 b c$ & $72.03 a$ & $23 a$ & $3203 a$ & $85.16 a$ & $8.76 \mathrm{~cd}$ \\
\hline & 0.05 & $28.66 b$ & $14.0 \mathrm{~b}$ & $388 c$ & $10.86 c$ & $10.78 a$ & $38.95 c$ & $18 b$ & $444 c$ & $19.13 c$ & $11.20 \mathrm{a}$ \\
\hline & 0.01 & $23.65 c$ & $12.1 \mathrm{c}$ & $221 c$ & $6.60 c$ & $11.31 \mathrm{a}$ & $31.36 d$ & $16 b$ & $416 c$ & $17.41 \mathrm{c}$ & 10.67ab \\
\hline \multirow[t]{3}{*}{ ANOVA } & $\mathrm{CO}_{2}$ & $*$ & ns & ns & $*$ & $*$ & $* * *$ & $*$ & $*$ & $* *$ & $* *$ \\
\hline & $\mathrm{Pi}$ & $* * *$ & $* * *$ & $* * *$ & $* * *$ & $* * *$ & $* * *$ & $* * *$ & $* * *$ & $* * *$ & $* * *$ \\
\hline & $\mathrm{CO}_{2} \times \mathrm{Pi}$ & ns & ns & ns & $*$ & ns & ns & ns & $*$ & $*$ & ns \\
\hline
\end{tabular}

Significant at $* \mathrm{P} \leq 0.05 ; * * \mathrm{P} \leq 0.01 ; * * * \mathrm{P} \leq 0.001$; and $\mathrm{ns}$, non-significant $(\mathrm{P}>0.05)$. Within columns for each experiment, means followed by same letters are not significantly different at $\alpha=0.05$. 


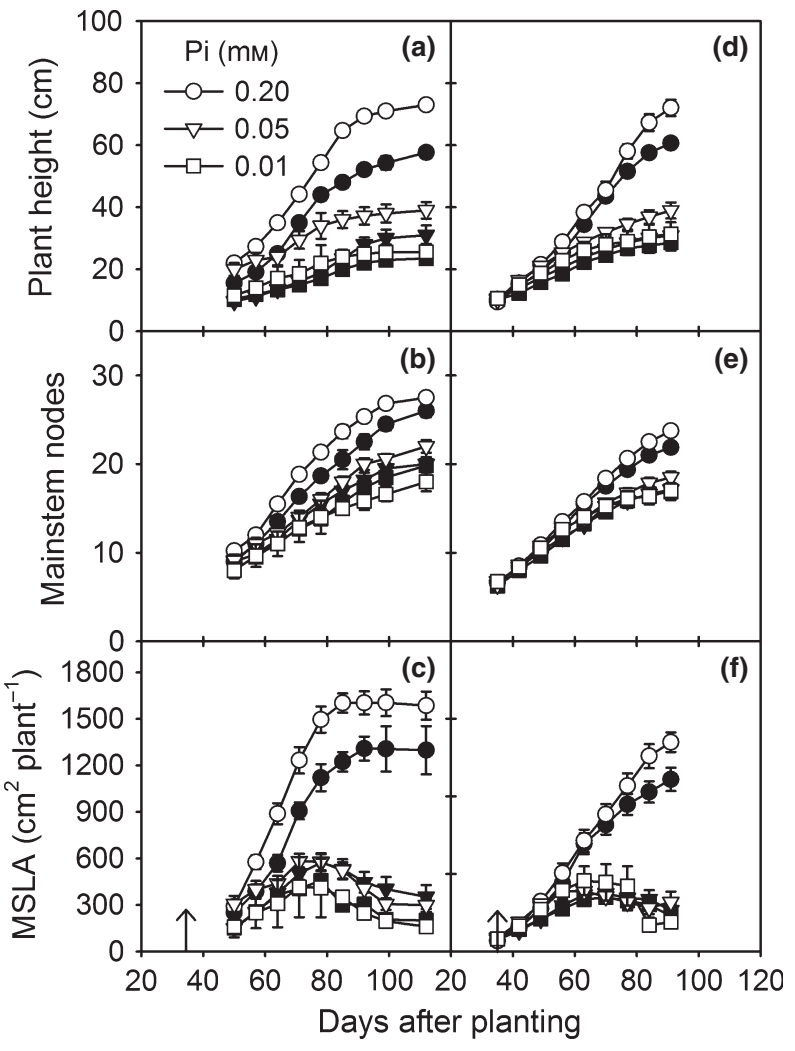

Fig. 1 Changes in plant height, number of main stem node (plant ${ }^{-1}$ ) and main stem leaf area (MSLA) for different phosphate-(Pi) treated cotton plants grown at either ambient (filled symbols, $400 \mu \mathrm{mol} \mathrm{mol}^{-1}$ ) or elevated (unfilled symbols, $800 \mu \mathrm{mol} \mathrm{mol}^{-1}$ ) $\mathrm{CO}_{2}$ for Experiments I (ac) and II (d-f). Treatments were initiated at zero $\left(\mathrm{CO}_{2}\right)$ and 34 (Pi, arrow) days after planting (DAP) in Exp. I, and both treatments were initiated at 34 DAP in Exp. II.

fraction of leaf, stem and fruit biomass to the total biomass tended to be lower under Pi-stress treatments $(0.05$ and $0.01 \mathrm{~mm} \mathrm{Pi}$ ), whereas fraction of root biomass to the total biomass increased up to threefold consistently across experiments (Table 2). Elevated $\mathrm{CO}_{2}$ significantly $(\mathrm{P}<0.01)$ increased the fraction of stem biomass to the total biomass in both experiments.

\section{Gas exchange measurements}

The $\mathrm{CO}_{2} \times \mathrm{Pi}$ interactions were significant $(\mathrm{P}<0.05)$ for $P_{\text {net }}$ and $g_{s}$, when averaged over measurement dates (Table 3). Plants grown under Pi-stress treatments (0.05 and $0.01 \mathrm{~mm}$ ) consistently exhibited lower $P_{\text {net }}$ for all measurement dates irrespective of $\mathrm{CO}_{2}$ treatments (Fig. 2a). Averaged across measurement dates, the severe Pi-stress treatment $(0.01 \mathrm{~mm})$ caused 68 and $77 \%$ reduction in $P_{\text {net }}$ at ambient and elevated $\mathrm{CO}_{2}$, respectively, as compared to $0.20 \mathrm{~mm} \mathrm{Pi}$ treatment (Table 3). Elevated $\mathrm{CO}_{2}$ caused
$>18 \%$ higher $P_{\text {net }}$ at 0.20 and $0.05 \mathrm{~mm} \mathrm{Pi}$, relative to the $400 \mu \mathrm{mol} \mathrm{mol}{ }^{-1} \mathrm{CO}_{2}$ treatment, whereas $P_{\text {net }}$ was $17 \%$ lower under elevated $\mathrm{CO}_{2}$ in the $0.01 \mathrm{~mm}$ Pi-treated plants. Averaged across $\mathrm{Pi}$ and $\mathrm{CO}_{2}$ treatments, $g_{\mathrm{s}}$ decreased by $33 \%$ at elevated $\mathrm{CO}_{2}$ and by $60 \%$ under Pi-stress. There was significant $(\mathrm{P}<0.05) \mathrm{CO}_{2} \times \mathrm{Pi}$ interaction for $\mathrm{C}_{\mathrm{i}} / \mathrm{C}_{\mathrm{a}}$ leading to relatively lower $\mathrm{C}_{\mathrm{i}} / \mathrm{C}_{\mathrm{a}}$ at $0.01 \mathrm{~mm} \mathrm{Pi}$ only at elevated $\mathrm{CO}_{2}$. The main effect of $\mathrm{Pi}$ was not significant $(\mathrm{P}>0.05)$ for $\mathrm{C}_{\mathrm{i}} / \mathrm{C}_{\mathrm{a}}$.

\section{Tissue constituents}

There was significant $\mathrm{CO}_{2} \times \mathrm{Pi}$ interaction for chlorophyll concentration (Table 3). Irrespective of $\mathrm{CO}_{2}$ levels, chlorophyll concentration decreased significantly $(\mathrm{P}<0.001)$ under Pi-stress treatments. The $\mathrm{P}, \mathrm{C}$ and $\mathrm{N}$ contents in uppermost fully expanded leaves were assessed on both leaf mass $\left(\mathrm{mg} \mathrm{g}^{-1}\right)$ and leaf area basis $\left(\mathrm{mg} \mathrm{cm}^{-2}\right)$. The $\mathrm{P}$ content (mass-based) in uppermost fully expanded leaves tended to be lower at most of the measurement dates in plants grown at either elevated $\mathrm{CO}_{2}$ or under Pi-stress treatments (Fig. 2b,c). When averaged across measurement dates, leaf $\mathrm{P}$ content of uppermost fully expanded leaves significantly $(\mathrm{P}<0.05)$ decreased under $\mathrm{Pi}$-stress treatments irrespective of the calculation methods (leaf massor leaf area-based) (Table 3). However, elevated $\mathrm{CO}_{2}$ significantly $(\mathrm{P}<0.05)$ decreased leaf mass-based $\mathrm{P}$ content but not leaf area-based $\mathrm{P}$ content (Table 3). Elevated $\mathrm{CO}_{2}$ significantly $(\mathrm{P}<0.05)$ affected $\mathrm{N}$ content leading to lower tissue $\mathrm{N}$, whereas effect of $\mathrm{Pi}$ was not significant $(\mathrm{P}>0.05)$. Irrespective of $\mathrm{Pi}$ treatments, $\mathrm{C}$ content (area basis) and $\mathrm{C}: \mathrm{N}$ ratio were significantly $(\mathrm{P}<0.05)$ higher at elevated $\mathrm{CO}_{2}$ in uppermost fully expanded leaves.

The overall nutrient composition in the cotton plant organs (leaves i.e. total from main stem and branches, stems and roots) for the final harvest in both experiments is shown in Table 4. The $\mathrm{CO}_{2} \times \mathrm{Pi}$ interactions for $\mathrm{P}$ contents were not significant $(\mathrm{P}>0.05)$ except for the stems in Exp. I. Pi-stress significantly $(\mathrm{P}<0.05)$ decreased $\mathrm{P}$ content in all plant organs, whereas the effect of $\mathrm{CO}_{2}$ was not consistent. On average, tissue $\mathrm{P}$ and $\mathrm{N}$ tended to be either slightly higher (leaf and stem) or lower (roots) in Exp. I vs. Exp. II. Stems appeared to have the lowest P and N contents in both experiments. The $\mathrm{N}$ content did not show significant $(\mathrm{P}>0.05) \mathrm{CO}_{2} \times \mathrm{Pi}$ interactions except in roots (Table 4). In general, the tissue $\mathrm{N}$ tended to be higher under Pi-stress and lower at elevated $\mathrm{CO}_{2}$ across plant organs.

\section{Relationship of tissue $\mathrm{P}$ with other parameters}

Regressions analysis revealed significant relationships between tissue $\mathrm{P}$ contents and all other parameters 
Table 2 Effect of $\mathrm{CO}_{2}$ levels ( $\mu \mathrm{mol} \mathrm{mol}{ }^{-1}$ ) and phosphate ( $\mathrm{Pi}, \mathrm{mm}$ ) supply on biomass partitioning of cotton plants at final harvests of Exp. I (112 DAP) and Exp. II (91 DAP). Analysis of variance (ANOVA) between Pi and $\mathrm{CO}_{2}$ is given. The data are the mean of six-eight individual plants

\begin{tabular}{|c|c|c|c|c|c|c|c|c|c|}
\hline \multirow[b]{2}{*}{$\mathrm{CO}_{2}$} & \multirow[b]{2}{*}{$\mathrm{Pi}$} & \multicolumn{4}{|c|}{ Tissue biomass (g plant ${ }^{-1}$ ) } & \multicolumn{4}{|c|}{ Fraction of total biomass (\%) } \\
\hline & & Leaves & Stems & Fruits & Roots & Leaves & Stems & Fruits & Roots \\
\hline \multicolumn{10}{|c|}{ Experiment I } \\
\hline \multirow[t]{3}{*}{400} & 0.20 & $29.83 b$ & $29.46 b$ & $34.30 a$ & $22.07 b$ & $26.02 b c$ & $25.64 b c$ & $29.22 a$ & $19.12 d$ \\
\hline & 0.05 & $12.06 \mathrm{c}$ & $7.29 c$ & $1.07 \mathrm{~b}$ & $8.95 c$ & $41.70 a$ & $24.59 c$ & $3.38 b$ & $30.33 c$ \\
\hline & 0.01 & $0.91 \mathrm{e}$ & $1.40 d$ & $0.28 b$ & $3.31 \mathrm{de}$ & $15.25 d$ & $24.30 c$ & $0.27 \mathrm{~b}$ & $60.19 a$ \\
\hline \multirow[t]{3}{*}{800} & 0.20 & 47.81a & $55.86 a$ & $41.27 a$ & $28.48 a$ & $27.77 b$ & $32.33 a b$ & $23.50 a$ & $16.41 d$ \\
\hline & 0.05 & $6.36 d$ & $5.39 \mathrm{~cd}$ & $5.91 b$ & $5.28 d$ & $28.81 b$ & $23.61 c$ & $24.84 a$ & $22.75 d$ \\
\hline & 0.01 & $0.81 \mathrm{e}$ & $1.07 d$ & $0.06 b$ & $1.47 \mathrm{e}$ & $18.38 \mathrm{~cd}$ & $35.70 a$ & $0.12 b$ & $45.92 b$ \\
\hline \multirow[t]{3}{*}{ ANOVA } & $\mathrm{CO}_{2}$ & $* * *$ & $* * *$ & ns & ns & ns & $* *$ & ns & $* * *$ \\
\hline & $\mathrm{Pi}$ & $* * *$ & $* * *$ & $* * *$ & $* * *$ & $* * *$ & ns & $* * *$ & $* * *$ \\
\hline & $\mathrm{CO}_{2} \times \mathrm{Pi}$ & $* * *$ & $* * *$ & ns & $* * *$ & $*$ & ns & $* * *$ & ns \\
\hline \multicolumn{10}{|c|}{ Experiment II } \\
\hline \multirow[t]{3}{*}{400} & 0.20 & $17.75 b$ & $19.60 \mathrm{~b}$ & $15.26 a$ & $9.84 a$ & $27.77 \mathrm{~b}$ & $30.54 b c$ & $24.12 \mathrm{a}$ & $15.15 d$ \\
\hline & 0.05 & $4.08 c$ & $4.08 c$ & $2.64 b$ & $4.61 b$ & $25.94 b c$ & $26.47 \mathrm{~cd}$ & $16.63 b$ & $30.96 b c$ \\
\hline & 0.01 & $3.29 c$ & $3.75 c$ & $3.02 b$ & $4.67 b$ & $22.38 c$ & $25.42 d$ & $15.46 b$ & $36.74 a b$ \\
\hline \multirow[t]{3}{*}{800} & 0.20 & $28.02 a$ & $30.88 a$ & $14.26 a$ & $12.01 \mathrm{a}$ & $32.98 a$ & $36.39 a$ & $16.53 b$ & $14.10 d$ \\
\hline & 0.05 & $4.99 c$ & $5.37 c$ & $3.80 \mathrm{~b}$ & $4.98 b$ & $24.65 b c$ & $28.48 \mathrm{bcd}$ & $19.81 a b$ & $27.06 \mathrm{c}$ \\
\hline & 0.01 & $4.27 c$ & $5.83 c$ & $1.17 \mathrm{~b}$ & $6.13 b$ & $22.97 c$ & $31.41 b$ & $3.47 c$ & $42.15 a$ \\
\hline \multirow[t]{3}{*}{ ANOVA } & $\mathrm{CO}_{2}$ & $* *$ & $* * *$ & ns & ns & ns & $* * *$ & $* *$ & ns \\
\hline & $\mathrm{Pi}$ & $* * *$ & $* * *$ & $* * *$ & $* * *$ & $* * *$ & $* * *$ & $* * *$ & $* * *$ \\
\hline & $\mathrm{CO}_{2} \times \mathrm{Pi}$ & $* *$ & $* *$ & ns & ns & ns & ns & $* *$ & ns \\
\hline
\end{tabular}

Significant at $* \mathrm{P} \leq 0.05 ; * * \mathrm{P} \leq 0.01 ; * * * \mathrm{P} \leq 0.001$; and $\mathrm{ns}=$ non-significant $(\mathrm{P}>0.05)$. Within columns for each experiment, means followed by same letters are not significantly different at $\alpha=0.05$.

Table 3 Effect of $\mathrm{CO}_{2}$ levels $\left(\mu \mathrm{mol} \mathrm{mol}{ }^{-1}\right.$ ) and phosphate $(\mathrm{Pi}, \mathrm{mM})$ supply on the rate of photosynthesis $\left(P_{\text {net, }} \mu \mathrm{mol} \mathrm{m} \mathrm{m}^{-2} \mathrm{~s}^{-1}\right)$, stomatal conductance $\left(g_{\mathrm{s}}, \mathrm{mol} \mathrm{H} \mathrm{H} \mathrm{m}^{-2} \mathrm{~s}^{-1}\right)$, internal $\left(\mathrm{C}_{\mathrm{i}}\right)$ to external $\left(\mathrm{C}_{\mathrm{a}}\right) \mathrm{CO}_{2}$ ratio $\left(\mathrm{C}_{\mathrm{i}} / \mathrm{C}_{\mathrm{a}}\right)$, specific leaf weight (SLW, $\mathrm{mg} \mathrm{cm}^{-2}$ of single leaf), total chlorophyll concentration $\left(\mathrm{Chl}, \mu \mathrm{g} \mathrm{cm}^{-2}\right)$, carbon $(\mathrm{C})$ and nitrogen $(\mathrm{N})$ ratio $(\mathrm{C} / \mathrm{N})$, tissue phosphorus $(\mathrm{P}), \mathrm{C}$, and $\mathrm{N}$ contents based on leaf mass (mg g $\mathrm{g}^{-1} \mathrm{dry}$ weight) and area $\left(\mathrm{mg} \mathrm{cm}^{-2}\right.$ area) of upper most fully expanded leaves of cotton. Analysis of variance (ANOvA) between Pi and $\mathrm{CO}_{2}$ is given. The data are mean of 13-day measurements between 57 and 112 days after planting across two experiments

\begin{tabular}{|c|c|c|c|c|c|c|c|c|c|c|c|c|c|}
\hline \multirow[b]{2}{*}{$\mathrm{CO}_{2}$} & \multirow[b]{2}{*}{$\mathrm{Pi}$} & \multirow[b]{2}{*}{$P_{\text {net }}$} & \multirow[b]{2}{*}{$g_{\mathrm{s}}$} & \multirow[b]{2}{*}{$C_{i} / C_{a}$} & \multirow[b]{2}{*}{ SLW } & \multirow[b]{2}{*}{$\mathrm{Chl}$} & \multirow[b]{2}{*}{$\mathrm{C} / \mathrm{N}$} & \multicolumn{3}{|c|}{$\mathrm{mg} \mathrm{g}^{-1}$ leaf dry weight } & \multicolumn{3}{|c|}{$\mathrm{mg} \mathrm{cm}^{-2}$ leaf area } \\
\hline & & & & & & & & $P$ & C & N & $P$ & C & N \\
\hline \multirow[t]{3}{*}{400} & 0.20 & $23.35 b$ & $0.35 a$ & $0.63 c$ & $9.79 c$ & $25.7 a$ & $13.4 c$ & $1.19 a$ & $428 a$ & $33.2 a$ & $0.0114 a$ & $4.17 d$ & $0.324 a b c$ \\
\hline & 0.05 & $11.07 d$ & $0.16 c$ & $0.64 b c$ & $10.29 b c$ & $10.0 \mathrm{c}$ & $13.7 c$ & $0.61 c$ & $438 a$ & $32.4 a$ & $0.0063 b$ & $4.51 d$ & $0.334 a b$ \\
\hline & 0.01 & 7.73e & $0.14 c$ & $0.69 a b$ & $10.65 b$ & $8.6 c$ & $13.8 \mathrm{c}$ & $0.54 c d$ & $433 a$ & $32.0 a$ & $0.0057 b$ & $4.64 c d$ & $0.340 a$ \\
\hline \multirow[t]{3}{*}{800} & 0.20 & $28.3 a$ & $0.25 b$ & $0.69 a b$ & $12.18 a$ & $29.5 b$ & $17.8 b$ & $0.92 b$ & $422 a$ & $24.2 b c$ & $0.0111 \mathrm{a}$ & $5.19 b$ & $0.297 b c$ \\
\hline & 0.05 & $13.12 \mathrm{c}$ & $0.14 c$ & $0.72 a$ & $11.97 a$ & $12.9 \mathrm{~cd}$ & $16.7 b$ & $0.51 \mathrm{~cd}$ & $424 a$ & $26.0 \mathrm{~b}$ & $0.0061 b$ & $5.06 \mathrm{bc}$ & $0.307 a b c$ \\
\hline & 0.01 & $6.37 e$ & $0.04 d$ & $0.67 a b c$ & $12.48 a$ & $7.2 \mathrm{~d}$ & $20.1 a$ & $0.49 d$ & $442 a$ & $22.3 c$ & $0.006 b$ & $5.71 a$ & $0.287 c$ \\
\hline \multirow[t]{3}{*}{ ANOVA } & $\mathrm{CO}_{2}$ & $* * *$ & $* * *$ & $*$ & $* * *$ & $* * *$ & $* * *$ & $* * *$ & ns & $* * *$ & ns & $* * *$ & $*$ \\
\hline & $\mathrm{Pi}$ & $* * *$ & $* * *$ & ns & ns & $* * *$ & $* *$ & $* * *$ & ns & ns & $* * *$ & $*$ & ns \\
\hline & $\mathrm{CO}_{2} \times \mathrm{Pi}$ & $* * *$ & $*$ & $*$ & ns & $*$ & $*$ & $*$ & ns & ns & ns & ns & ns \\
\hline
\end{tabular}

Significant at $* \mathrm{P} \leq 0.05 ; * * \mathrm{P} \leq 0.01 ; * * \mathrm{P} \leq 0.001$; and $\mathrm{ns}=$ non-significant $(\mathrm{P}>0.05)$. Within columns, means followed by same letters are not significantly different at $\alpha=0.05$.

$(\mathrm{P}<0.05)$ (Figs 3-6), except tissue $\mathrm{N}$ content of the uppermost fully expanded leaves (Fig. 6b). The slope of the linear regression between $\mathrm{CO}_{2}$ levels differed significantly $(\mathrm{P}<0.05)$ for whole plant tissue $\mathrm{P}$ content vs. TBM measured at the final harvest across experiments (Fig. 3a). Elevated $\mathrm{CO}_{2}$ exhibited more biomass accumulation and the differences between elevated vs. ambient $\mathrm{CO}_{2}$ tended to increase with increase in tissue $\mathrm{P}$ content. The relationship between whole plant tissue $\mathrm{P}$ content and root:shoot ratio was nonlinear and did not differ significantly $(\mathrm{P}>0.05)$ between $\mathrm{CO}_{2}$ treatments (Fig. 3b). The root:shoot ratio increased with decrease in tissue $\mathrm{P}$ content. A curvilinear 


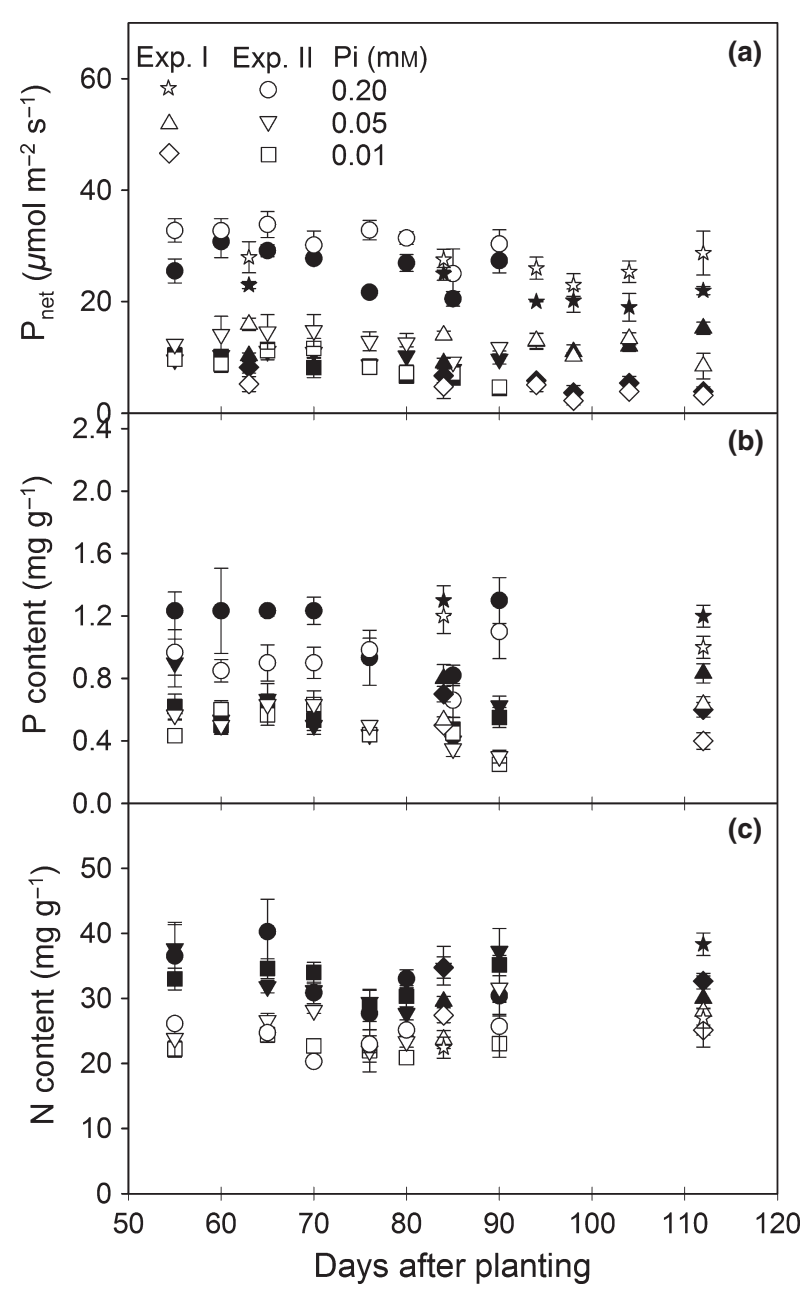

Fig. 2 Photosynthetic rate $\left(P_{\text {net }}\right)$, phosphorus $(\mathrm{P})$ and nitrogen $(\mathrm{N})$ contents of dry tissue for upper most fully expanded cotton leaves during experiment grown at either ambient (filled symbols, $400 \mu \mathrm{mol} \mathrm{mol}^{-1}$ ) or elevated (unfilled symbols, $800 \mu \mathrm{mol} \mathrm{mol}^{-1}$ ) $\mathrm{CO}_{2}$ under different phosphate (Pi) treatments. The data are from two experiments (Exps I and II). The error bars represent the standard error of 3-5 individual plants.

(polynomial second order) relationship was observed between mass- and area-based tissue $\mathrm{P}$ content in uppermost fully expanded leaves (Fig. 4). The linear term between $\mathrm{CO}_{2}$ levels differed $(\mathrm{P}<0.05)$ for mass- vs. areabased $\mathrm{P}$ curve. For the two levels of $\mathrm{CO}_{2}$ treatments, the slope of the regression between tissue $\mathrm{P}$ content (both mass- and area-based) and $P_{\text {net }}$ of uppermost fully expanded leaves differed significantly $(\mathrm{P}<0.05)$ (Fig. 5a, b). The $g_{s}$ response to leaf $\mathrm{P}$ content between $\mathrm{CO}_{2}$ levels differed only when tissue $\mathrm{P}$ content was expressed on leaf area basis (Fig. 5c,d). Chlorophyll concentration also increased with leaf $\mathrm{P}$ content $\left(\mathrm{mg} \mathrm{g}^{-1}\right)$ and did not differ significantly $(\mathrm{P}>0.05)$ between $\mathrm{CO}_{2}$ treatments (Fig. 6a). A significant relationship between leaf $\mathrm{P}$ and $\mathrm{N}$ contents was not established; however, $\mathrm{N}$ tended to be higher at ambient vs. elevated $\mathrm{CO}_{2}$ at a given leaf $\mathrm{P}$ content (Fig. 6b).

\section{Discussion}

\section{Growth and development}

The patterns of cotton growth responses to the treatments ( $\mathrm{Pi}$ and $\mathrm{CO}_{2}$ ) were similar in both experiments. However, they differed in magnitude possibly due to the differences in initiation of $\mathrm{CO}_{2}$ treatment and time of harvests between Exp. I and Exp. II. Despite some interaction between treatments mostly in Exp. I, the growth and development responses to Pi supply were similar across both $\mathrm{CO}_{2}$ levels. The observed stunted plants with fewer main stem nodes and decreased leaf area under Pi-stress treatments were consistent with the observations made in other nutrient studies (Reddy and Zhao 2005, Fleisher et al. 2012). Smaller plant stature under Pi-stress resulted in over $77 \%$ lower biomass accumulation across $\mathrm{CO}_{2}$ treatments. Radin and Eidenbock (1984) reported that phosphate deficiency limited cell expansion by reducing hydraulic conductance inside plants, which may lead to reduced plant size and leaf area expansion. This was supported by severely decreased leaf area under Pi-stress treatments in the current study. In fact, main stem leaf area began to decline under Pi-stress roughly 75 DAP in both experiments due to leaf senescence. Phosphate deficiency-induced early leaf senescence has also been suggested in potato (Fleisher et al. 2013). The plant dry weight and total leaf area followed similar trend across the treatments at the first harvest in both the experiments; however, it varied in the final harvest particularly under Pi-stress due to leaf senescence. Elevated $\mathrm{CO}_{2}$ significantly stimulated stem elongation, leaf area expansion and total biomass at $0.20 \mathrm{~mm}$ Pi treatments; however, this stimulation was rarely significant for $0.05 \mathrm{~mm} \mathrm{Pi}$ and none for $0.01 \mathrm{~mm} \mathrm{Pi}$ treatments suggesting unresponsiveness of these traits to $\mathrm{CO}_{2}$ enrichment under Pi-stress treatments.

\section{Biomass partitioning}

The observed decrease (Pi-stress treatments) or increase (elevated $\mathrm{CO}_{2}$, especially at higher Pi supply) in biomass accumulation of plant organs was in accordance with other studies and attributed to the adjustment of plant growth under nutrient deficiency and $\mathrm{CO}_{2}$ enrichment (Kimball and Mauney 1993, Reddy and Zhao 2005, Fleisher et al. 2012, Lenka and Lal 2012). In spite of an increased fruit and root biomass at elevated $\mathrm{CO}_{2}$ under full nutrient supply $(0.20 \mathrm{~mm} \mathrm{Pi})$, the main effect of elevated $\mathrm{CO}_{2}$ on fruit and root biomass was not significant. The fruit abscission was affected more under elevated $\mathrm{CO}_{2}$ than under ambient 
Table 4 Effect of $\mathrm{CO}_{2}$ levels $\left(\mu \mathrm{mol} \mathrm{mol} \mathrm{m}^{-1}\right.$ ) and phosphate ( $\mathrm{Pi}, \mathrm{mm}$ ) supply on phosphorus (P) and nitrogen ( $\mathrm{N}$ ) content in tissues of cotton plant and its organs (leaves, stems and roots). Analysis of variance (ANOVA) between Pi and $\mathrm{CO}_{2}$ is given. Data are from six-eight individual plants at the final harvests of the two experiments

\begin{tabular}{|c|c|c|c|c|c|c|c|c|c|}
\hline \multirow[b]{2}{*}{$\mathrm{CO}_{2}$} & \multirow[b]{2}{*}{$\mathrm{Pi}$} & \multicolumn{4}{|c|}{$P\left(\mathrm{mg} \mathrm{g}^{-1}\right)$} & \multicolumn{4}{|c|}{$\mathrm{N}\left(\mathrm{mg} \mathrm{g}^{-1}\right)$} \\
\hline & & Plant & Leaves & Stems & Roots & Plant & Leaves & Stems & Roots \\
\hline \multicolumn{10}{|c|}{ Experiment I } \\
\hline \multirow[t]{3}{*}{400} & 0.20 & $1.05 a$ & $1.15 a$ & $0.83 a$ & $1.07 a$ & $27.5 b$ & $45.0 \mathrm{~b}$ & $20.6 b$ & $20.2 a$ \\
\hline & 0.05 & $0.88 b$ & $0.80 \mathrm{~b}$ & $0.97 a$ & $0.92 \mathrm{ab}$ & $34.1 a$ & $52.3 a$ & $20.5 b$ & $20.2 a$ \\
\hline & 0.01 & $0.54 c$ & $0.87 b$ & $0.53 b$ & $0.62 c$ & $29.7 \mathrm{~cd}$ & $53.0 a$ & $22.0 a$ & 18.9ab \\
\hline \multirow[t]{3}{*}{800} & 0.20 & $0.78 b$ & $0.82 b$ & $0.58 b$ & $0.92 a b$ & 22.6de & $34.0 c$ & $15.7 c$ & $16.7 b$ \\
\hline & 0.05 & $0.65 c$ & $0.60 c$ & $0.62 b$ & $0.78 b c$ & $28.2 b c$ & $45.6 b$ & $17.9 b c$ & $18.4 \mathrm{ab}$ \\
\hline & 0.01 & $0.60 c$ & $0.68 b c$ & $0.50 b$ & $0.60 c$ & $24.0 \mathrm{e}$ & $45.0 \mathrm{~b}$ & $20.6 b$ & $20.2 a$ \\
\hline \multirow[t]{3}{*}{ ANOVA } & $\mathrm{CO}_{2}$ & $* * *$ & $* * *$ & $* * *$ & ns & $* * *$ & $* * *$ & $* * *$ & ns \\
\hline & $\mathrm{Pi}$ & $* * *$ & $* * *$ & $* * *$ & $* * *$ & $* * *$ & $* * *$ & ns & ns \\
\hline & $\mathrm{CO}_{2} \times \mathrm{Pi}$ & ns & ns & $*$ & ns & ns & ns & ns & $*$ \\
\hline \multicolumn{10}{|c|}{ Experiment II } \\
\hline \multirow{2}{*}{400} & 0.05 & $0.61 b$ & $0.60 b$ & $0.31 d$ & $0.88 b c$ & $30.4 a$ & $38.6 a$ & $16.7 a$ & $35.8 a$ \\
\hline & 0.01 & $0.59 b$ & $0.63 b$ & $0.38 \mathrm{~cd}$ & $0.73 \mathrm{~cd}$ & $28.4 a b$ & $38.0 a$ & $17.7 a$ & $30.2 a b$ \\
\hline \multirow[t]{3}{*}{800} & 0.20 & $0.94 a$ & $1.00 a$ & $0.61 a$ & $1.23 a$ & $21.5 d$ & $28.2 \mathrm{c}$ & 14.9ab & 33.0ab \\
\hline & 0.05 & $0.61 b$ & $0.60 \mathrm{~b}$ & $0.39 \mathrm{~cd}$ & $0.86 c$ & $23.0 \mathrm{~cd}$ & $32.1 b c$ & $18.6 a$ & $21.9 \mathrm{~cd}$ \\
\hline & 0.01 & $0.56 b$ & $0.59 b$ & $0.45 b c$ & $0.63 d$ & $22.0 d$ & $28.0 c$ & $17.3 a$ & $17.7 d$ \\
\hline \multirow[t]{3}{*}{ ANOVA } & $\mathrm{CO}_{2}$ & ns & ns & $* *$ & ns & $* * *$ & $* * *$ & ns & $* *$ \\
\hline & $\mathrm{Pi}$ & $* * *$ & $* * *$ & $* * *$ & $* * *$ & $*$ & ns & $* *$ & $*$ \\
\hline & $\mathrm{CO}_{2} \times \mathrm{Pi}$ & ns & ns & ns & ns & ns & ns & ns & $* * *$ \\
\hline
\end{tabular}

Significant at $* \mathrm{P} \leq 0.05 ; * * \mathrm{P} \leq 0.01 ; * * * \mathrm{P} \leq 0.001$; and $\mathrm{ns}=$ non-significant $(\mathrm{P}>0.05)$. Within columns for each experiment, means followed by same letters are not significantly different at $\alpha=0.05$.

due to Pi-stress, reversing the positive effect of $\mathrm{CO}_{2}$ enrichment observed under full nutrient supply. This might have caused a non-significant effect of elevated $\mathrm{CO}_{2}$ on fruit. Similarly, at elevated $\mathrm{CO}_{2}$, the root biomass was either reduced (Exp. I) or showed minor increase (Exp. 2) in $\mathrm{Pi}$-stressed plants resulting in a lack of overall significant effect of elevated $\mathrm{CO}_{2}$ on root biomass. Fleisher et al. (2013) also reported non-significant effect of elevated $\mathrm{CO}_{2}$ on potato root biomass. Under Pi-stress, the increased biomass partitioning to the roots was similar to trends previously observed in cotton and potato (Radin and Eidenbock 1984, Fleisher et al. 2012). Increased biomass partitioning to the roots under Pi-stress may be a mechanism to exploit below-ground resources in an effort to supply plant demand for nutrients such as phosphorus. Similarly, under elevated $\mathrm{CO}_{2}$, increased above-ground growth is often associated with increased root biomass thus, indicating adjustment between above- and below-ground plant growths (Lenka and Lal 2012). Severe fruit abscission under Pi-stress may also reduce the fraction of above-ground biomass and increase the fraction of root biomass contributing to total biomass. In field studies with cotton, Mullins and Burmester (1990) found highest biomass partitioning towards the fruiting structures, followed by leaves and then stem. Because the experiments were terminated well before plant maturity, the contribution of fruits to total biomass was lower under the highest Pi supply except for ambient $\mathrm{CO}_{2}$ in Exp. I.

\section{Gas exchange measurements}

Significant reductions in $P_{\text {net }}$ under Pi-stress have been reported in other studies (Barrett and Gifford 1995, Fleisher et al. 2012). Although, $g_{\mathrm{s}}$ reduced more than $P_{\text {net }}$ under low $\mathrm{Pi}$, it did not appear to be the main cause of photosynthetic limitation as deduced from an insignificant effect of $\mathrm{Pi}$ on $\mathrm{C}_{\mathrm{i}} / \mathrm{C}_{\mathrm{a}}$. Similar results have also been reported in other nutrient studies (Zhao et al. 2001, Jin et al. 2011). However, the minor decrease in $P_{\text {net }}$ at elevated vs. ambient $\mathrm{CO}_{2}$ under lowest $\mathrm{Pi}$ treatment (0.01 mM) might also suggest critical role of stomatal limitation to photosynthesis. The extremely low $g_{s}$ (0.04 mol $\left.\mathrm{H}_{2} \mathrm{O} \mathrm{m}^{-2} \mathrm{~s}^{-1}\right)$ under this particular situation might have reduced $\mathrm{C}_{\mathrm{i}}$ leading to lower $\mathrm{C}_{\mathrm{i}} / \mathrm{C}_{\mathrm{a}}$ ratio, thus the observed $\mathrm{CO}_{2} \times \mathrm{Pi}$ interaction. An increase in $\mathrm{C}_{\mathrm{i}}$ (thus $\mathrm{C}_{\mathrm{i}} / \mathrm{C}_{\mathrm{a}}$ ratio, as observed under Pi-stress across both $\mathrm{CO}_{2}$ except the $0.01 \mathrm{~mm} \mathrm{Pi}$ under elevated $\mathrm{CO}_{2}$ ) has often been associated with the onset of mesophyll/biochemical limitation to photosynthesis that highly depends on the severity of the stress (Brodribb 1996, Singh and 


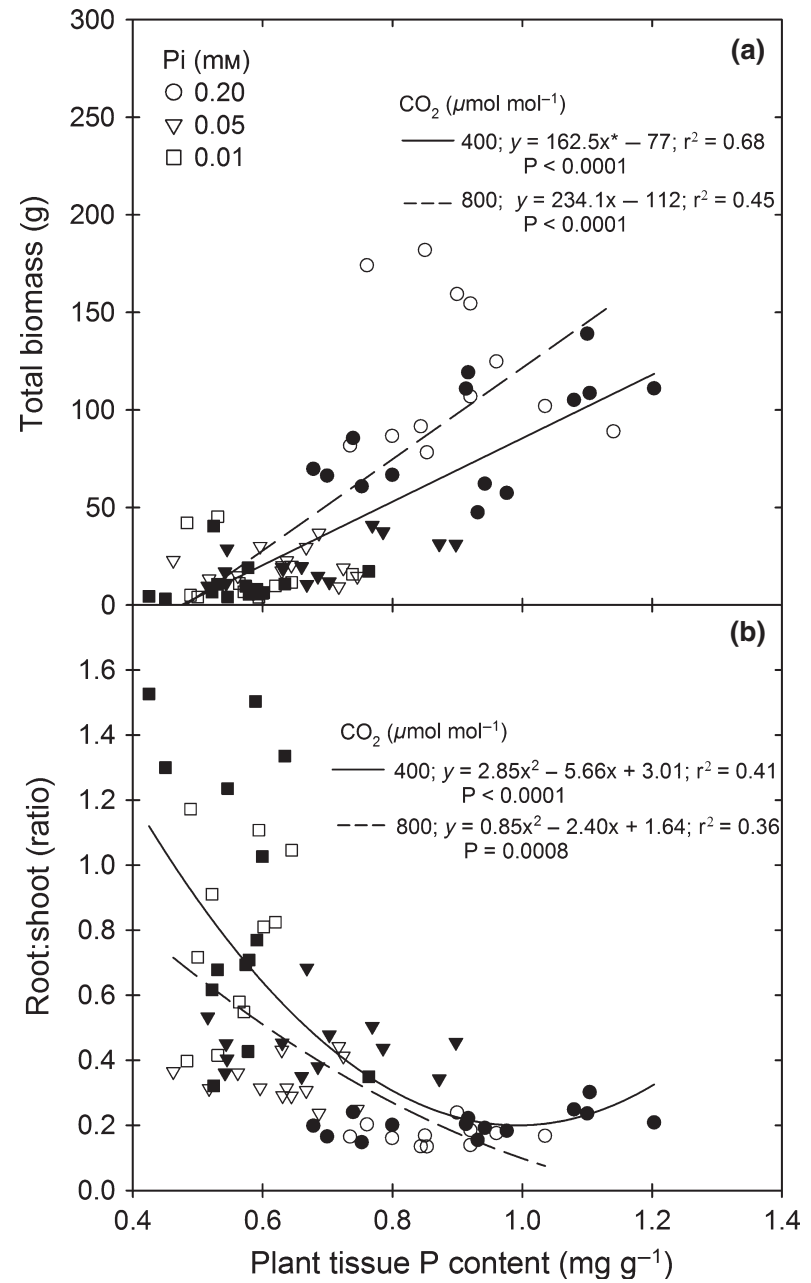

Fig. 3 Relationship between plant phosphorus (P) content and (a) total biomass and (b) root:shoot ratio of cotton grown at either ambient (filled symbols, $400 \mu \mathrm{mol} \mathrm{mol}^{-1}$ ) or elevated (unfilled symbols, $\left.800 \mu \mathrm{mol} \mathrm{mol}^{-1}\right) \mathrm{CO}_{2}$ under different phosphate (Pi) treatments. The slope (a) between $\mathrm{CO}_{2}$ differed significantly $(\mathrm{P}<0.05)$ as designated by an asterisk (*). Data are from individual plants at the final harvest of both experiments.

Reddy 2011). Therefore, the major influence of Pi on $P_{\text {net }}$ can still be attributed to factors other than $g_{\mathrm{s}}$ such as mesophyll resistance and inhibition of photo-biochemical processes as suggested in other studies (Longstreth and Nobel 1980, Jacob and Lawlor 1991, Fleisher et al. 2012). Jacob and Lawlor (1991) also found that relative stomatal limitation did not vary due to phosphorus nutrition but mesophyll limitation was greatly enhanced under Pi-stress across species. The severe reduction in chlorophyll concentration in the current study also suggested that metabolic inhibition of photosynthesis had occurred under Pi-stress. Enhancement of $P_{\text {net }}$ by elevated $\mathrm{CO}_{2}$ was greater at $0.20 \mathrm{~mm}$ vs. $0.05 \mathrm{~mm} \mathrm{Pi}$ and none for severe Pi-stress $(0.01 \mathrm{~mm})$ indicating reduction or lack in

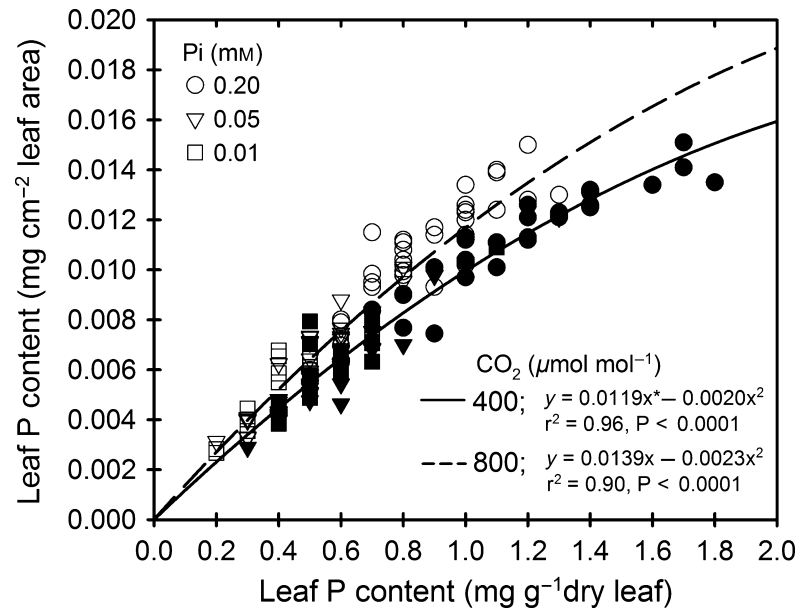

Fig. 4 Relationship between mass ( $\mathrm{mg} \mathrm{g}^{-1}$ leaf dry weight) and area bases ( $\mathrm{mg} \mathrm{cm}^{-2}$ leaf area) tissue P content in uppermost fully expanded cotton leaves grown at either ambient (filled symbols, $400 \mu \mathrm{mol} \mathrm{mol}^{-1}$ ) or elevated (unfilled symbols, $800 \mu \mathrm{mol} \mathrm{mol}^{-1}$ ) $\mathrm{CO}_{2}$ under different phosphate (Pi) treatments. Data are from individual plants measured between 57 and 112 days after planting across two experiments. A polynomial second order equation was used, and intercept was forced to pass through origin (zero). The asterisk $(*)$ indicated significant $(\mathrm{P}<0.05)$ difference in the linear term of the equations between two $\mathrm{CO}_{2}$ levels.

the stimulatory effect of elevated $\mathrm{CO}_{2}$ on photosynthetic response of plants as Pi-stress increased. Although tissue $\mathrm{P}$ content $\left(\mathrm{mg} \mathrm{g}^{-1}\right)$ of the uppermost fully expanded leaves was significantly lower at elevated vs. ambient $\mathrm{CO}_{2}$, this study suggested that it was not associated with downregulation of $P_{\text {net }}$ under elevated $\mathrm{CO}_{2}$ in Pi-stressed plants (Barrett and Gifford 1995). This might be due to the fact that the $\mathrm{P}$ content on a leaf area basis $(\mathrm{mg}$ $\mathrm{cm}^{-2}$ ) did not differ between $\mathrm{CO}_{2}$ treatments. Plant leaf nutrient status may provide a stable estimate based on leaf area as suggested in other studies (Bélanger et al. 2011). The increased $C$ content when estimated based on leaf area $\left(\mathrm{mg} \mathrm{cm}^{-2}\right)$ may be caused by restricted carbon export under Pi-stress, and increased carbon accumulation under elevated $\mathrm{CO}_{2}$ as also suggested by Radin and Eidenbock (1986).

\section{Tissue constituents}

In the uppermost fully expanded leaves, the observed reduction in the tissue $\mathrm{P}$ content without a significant effect on $\mathrm{N}$ content under Pi-stress treatments was comparable to the results in Pi deficient potato (Fleisher et al. 2012). Additionally, reduction in leaf tissue $\mathrm{P}$ and $\mathrm{N}$ contents under elevated $\mathrm{CO}_{2}$ is often reported similar to the observation made in this study (Rogers et al. 1993, Taub and Wang 2008, Lenka and Lal 2012). However, P content did 

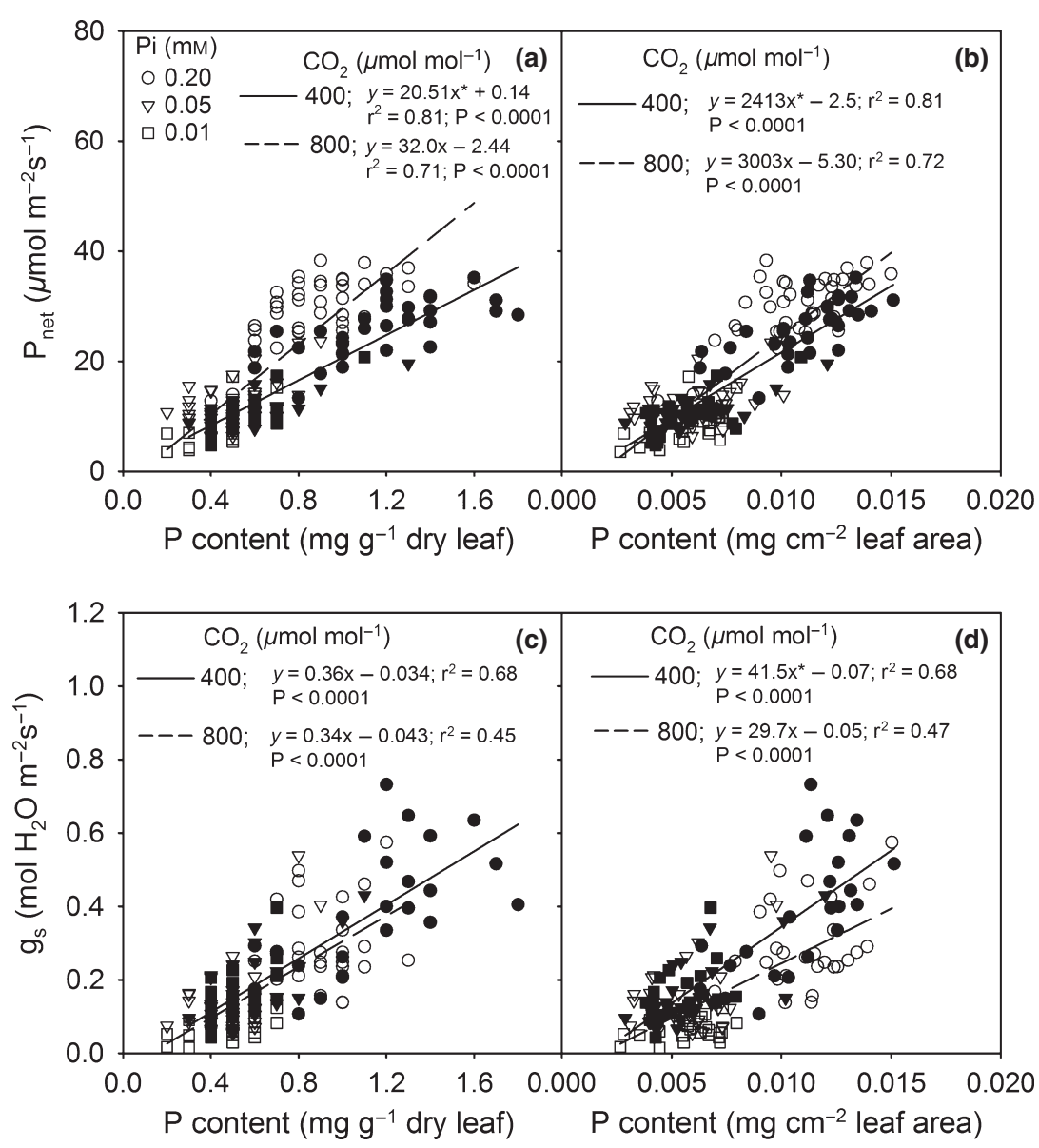

Fig. 5 Relationship of phosphorus $(P)$ content with photosynthetic rate $\left(P_{\text {net }}\right)(a, b)$ and stomatal conductance $\left(g_{s}\right)(c, d)$ for uppermost fully expanded cotton leaves grown at either ambient (filled symbols, $400 \mu \mathrm{mol} \mathrm{mol}^{-1}$ ) or elevated (unfilled symbols, $800 \mu \mathrm{mol} \mathrm{mol}{ }^{-1}$ ) $\mathrm{CO}_{2}$ under different phosphate (Pi) treatments. Data are from individual plants measured between 57 and 112 days after planting across two experiments. The asterisk (*) indicated significant $(\mathrm{P}<0.05)$ difference in the slope between two $\mathrm{CO}_{2}$ levels. not differ between $\mathrm{CO}_{2}$ levels in uppermost fully expanded leaves when expressed on leaf area basis $\left(\mathrm{mg} \mathrm{cm}^{-2}\right)$ indicating the dilution of the nutrients (e.g. $\mathrm{P}$ and $\mathrm{N}$ ) due to increased leaf thickness (e.g. SLW) and carbon accumulation under elevated $\mathrm{CO}_{2}$ (Taub and Wang 2008, Lenka and Lal 2012). This was also supported by increased C:N ratio of cotton leaves. Specific leaf weight signifies leaf thickness and similar to the current study has also been reported to increase in response to elevated $\mathrm{CO}_{2}$ in a wide range of species (Gifford et al. 2000).

Tissue $\mathrm{P}$ content of plant organs decreased under Pi-stress, but the differences between 0.05 and $0.01 \mathrm{~mm}$ $\mathrm{Pi}$ treatments were mostly not significant. Compared with other major nutrients, plants require phosphorus in smaller amounts and the low Pi treatments may not have shown large differences in the tissue $\mathrm{P}$ content, yet plant growth parameters largely differed. The observed lower nutrient content of stems compared with other plant organs may be explained by the high mobility of $\mathrm{N}$ and P (Prior et al. 1998). Mullins and Burmester (1990) also reported lower shoot $\mathrm{N}$ and $\mathrm{P}$ contents as compared with leaves and fruiting structures of cotton. The $\mathrm{N}$ content often increased under Pi-stress across plant organs indicating greater $\mathrm{N}$ uptake, as also suggested in earlier studies (Almeida et al. 2000, Fleisher et al. 2012). In the current study, the increased root:shoot ratio was due to increased biomass partitioning to the roots under Pi-stress suggesting relatively higher root volume might have assisted in the uptake of other nutrients such as $\mathrm{N}$. Under Pi-stress, the trend in tissue $\mathrm{P}$ content of plant organs was consistent with the uppermost fully expanded leaves; however, the variable results with respect to $\mathrm{CO}_{2}$ treatments were similar to the inconsistencies reported in other studies (Prior et al. 1998, 2003, Gifford et al. 2000). Under $\mathrm{CO}_{2}$ treatments, the variability in tissue $\mathrm{P}$ content between upper most fully expanded leaves and whole plant leaves might have partly been due to the fact that later consisted all the leaves from main stem and branches. The reductions in plant tissue $\mathrm{P}$ and $\mathrm{N}$ under elevated $\mathrm{CO}_{2}$ across $\mathrm{Pi}$ treatments have been reported by others; however, large variability also existed (Prior et al. 1998, 2003, Gifford et al. 2000). Elevated $\mathrm{CO}_{2}$ also exhibited a relatively consistent response by decreasing $\mathrm{N}$ content across plant components, except the roots in both experiments. This was also supported by the lack of $\mathrm{CO}_{2} \times \mathrm{Pi}$ interaction for tissue $\mathrm{N}$ content. The lack of $\mathrm{CO}_{2} \times \mathrm{Pi}$ 

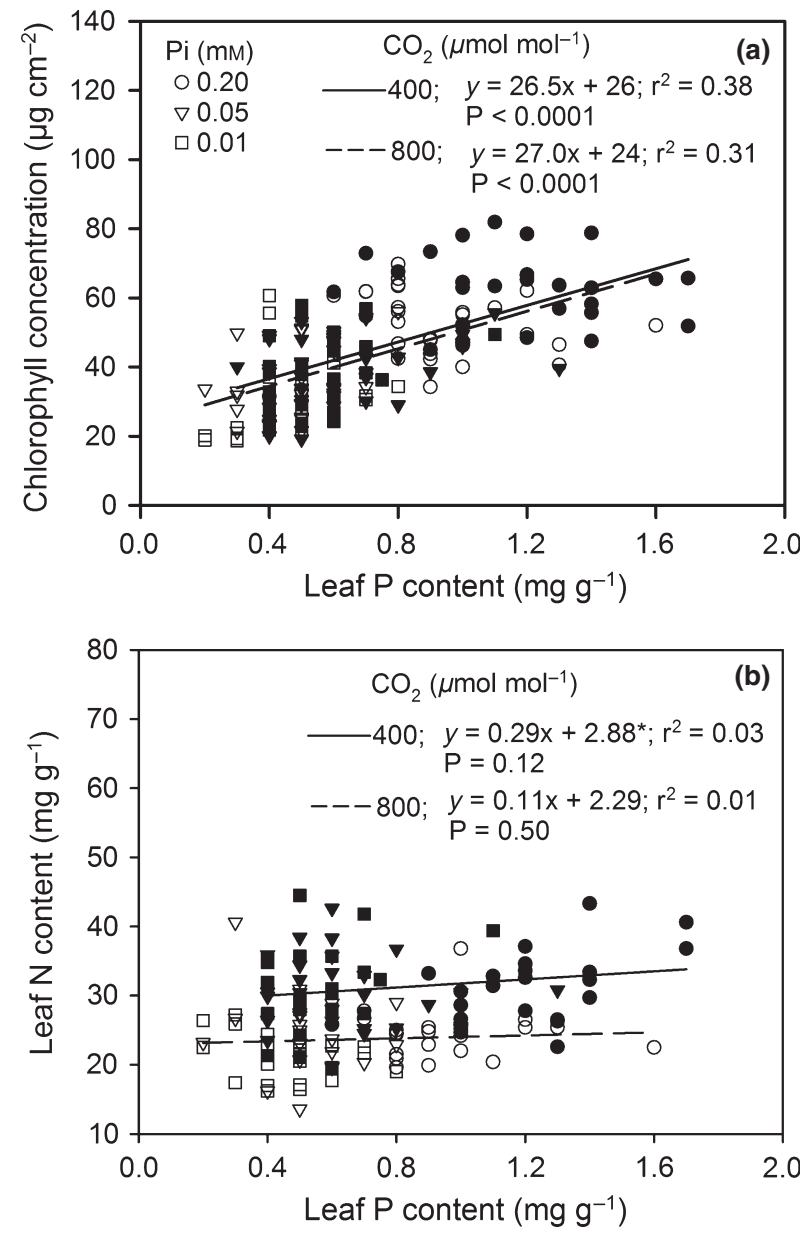

Fig. 6 Relationships of phosphorus (P) content with chlorophyll concentration (a) and nitrogen ( $\mathrm{N}$ ) content (b) for uppermost fully expanded cotton leaves grown at either ambient (filled symbols, $400 \mu \mathrm{mol} \mathrm{mol}^{-1}$ )

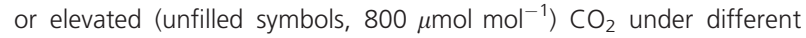
phosphate (Pi) treatments. Data are from individual plants measured between 57 and 112 days after planting across two experiments. The asterisk $(*)$ indicated significant $(P<0.05)$ difference in the intercept between two $\mathrm{CO}_{2}$ levels in (b).

interaction has also been reported in several potato organs (Fleisher et al. 2013). Although not clearly understood, factors such as dilution of $\mathrm{N}$ due to increased carbon assimilation, decreased $\mathrm{N}$ demand and restricted uptake and lower transpiration due to decrease in $g_{s}$ may contribute to the lower tissue $\mathrm{N}$ at elevated $\mathrm{CO}_{2}$ (Gifford et al. 2000, Taub and Wang 2008). The current study also suggested the existence of a dilution effect and lower transpiration as deduced from higher leaf thickness (SLW) and decreased $g_{s}$ under elevated $\mathrm{CO}_{2}$. This clearly indicated alteration in the uptake and utilization of $\mathrm{N}$, and lower $\mathrm{N}$ content under elevated $\mathrm{CO}_{2}$ might influence nitrateassimilation and biosynthesis of organic compounds (Bloom et al. 2010, Kawakami et al. 2012).

\section{Relationships between plant parameters and tissue $\mathrm{P}$ content}

Regardless of the $\mathrm{CO}_{2}$ levels, a linear increase in TBM, $P_{\text {net }}, g_{s}$ and chlorophyll concentration with tissue $\mathrm{P}$ content suggested that the highest Pi treatments of the current study did not reflect excessive Pi supply. The magnitude of the response of total biomass and $P_{\text {net }}$ to the tissue $\mathrm{P}\left(\mathrm{mg} \mathrm{g}^{-1}\right)$ differed between the two levels of $\mathrm{CO}_{2}$, which was in accordance with the significant $\mathrm{CO}_{2}$ effect observed for these parameters across Pi nutrition. Moreover, elevated $\mathrm{CO}_{2}$ increased $P_{\text {net }}$ response to leaf $\mathrm{P}$ content regardless of $\mathrm{P}$ calculation methods (mass vs. area). Studies reporting similar relationships between a range of leaf tissue $\mathrm{P}$ content (mass- vs. area-based) or with biomass and photosynthetic processes are limited. Reddy and Zhao (2005) found that the critical leaf potassium content varied between $\mathrm{CO}_{2}$ treatments for canopy photosynthesis and biomass production. The curvilinear relationships between mass- and area-based $\mathrm{P}$ contents across $\mathrm{CO}_{2}$ levels for uppermost fully expanded leaves delineated the subtle differences between expressions of nutrient content in leaves. For example, the difference in the coefficient of the linear regression (slope) of $P_{\text {net }}$ vs. leaf $\mathrm{P}$ content was smaller between ambient and elevated $\mathrm{CO}_{2}$ when $\mathrm{P}$ was expressed on leaf area basis than on a leaf mass basis (Fig. 5b). Similarly, compared with ambient $\mathrm{CO}_{2}$, the significantly lower regression slope between $g_{\mathrm{s}}$ and leaf $\mathrm{P}$ under elevated $\mathrm{CO}_{2}$ was only observed when $\mathrm{P}$ was expressed on an area basis $\left(\mathrm{mg} \mathrm{cm}^{-2}\right)$. Reduction in $g_{\mathrm{s}}$ in response to elevated $\mathrm{CO}_{2}$ is a well-known phenomenon, which was also observed in the current study across Pi supply (Ainsworth and Rogers 2007). Because the overall $P_{\text {net }}, g_{\mathrm{s}}$ or light interception are based on leaf area, it may be suitable to express leaf nutrient content on area basis for determining their relationships. The parallel response of chlorophyll concentration to tissue $\mathrm{P}$ for the two level of $\mathrm{CO}_{2}$ was consistent with the potassium nutrition study in cotton by Reddy and Zhao (2005). Although, there was not a clear relationship between leaf tissue $\mathrm{N}$ and $\mathrm{P}$ contents, $\mathrm{N}$ tended to be lower at elevated $\mathrm{CO}_{2}$ across the range of tissue $\mathrm{P}$ content. The nutrient dilution in plant tissue due to enhanced growth and decreased nutrient uptake may contribute to the lower tissue $\mathrm{N}$ at elevated $\mathrm{CO}_{2}$. However, more studies are needed to confirm the underlying physiological mechanisms (Taub and Wang 2008).

In summary, a substantial decrease in plant biomass under Pi deficiency was attributed to stunted growth primarily due to decreased leaf area accompanied with early leaf senescence and reduced photosynthesis. Consequently, Pi deficiency significantly shifted biomass partitioning by decreasing leaf and fruit production and increasing the root 
fraction of total biomass. Cotton growth and photosynthesis increased with tissue P content. Furthermore, the plant tissue $\mathrm{N}$ content tended to increase under Pi-stress. The increased fraction of root biomass under Pi-stress might have enhanced $\mathrm{N}$ uptake leading to higher tissue $\mathrm{N}$ content. The $\mathrm{CO}_{2} \times \mathrm{Pi}$ interactions were dependent on plant organs and type of growth processes measured. The growth enhancement effects of elevated $\mathrm{CO}_{2}$ were either highly reduced or trivial under the studied range of Pi-stress treatments. As a consequence, several processes such as number of main stem nodes and leaf area development, fruit set, photosynthesis, chlorophyll concentration were negatively affected by elevated $\mathrm{CO}_{2}$ under severe Pi-stress $(0.01 \mathrm{~mm})$. Despite the lower leaf $\mathrm{P}$ and stomatal conductance at elevated $\mathrm{CO}_{2}$, the contribution of these factors to the inhibition of growth and photosynthetic response at elevated $\mathrm{CO}_{2}$ under severe Pi-stress treatments were insignificant. A significant reduction in tissue $\mathrm{N}$ content (up to $21 \%$ in plant, averaged across $\mathrm{Pi}$ supply) under elevated $\mathrm{CO}_{2}$ appears to be critical and may need more attention to understand its influence on the overall effect of $\mathrm{CO}_{2}$ enrichment on plants.

\section{References}

Ahmad, Z., M. A. Gill, R. H. Qureshi, H. Rehman, and T. Mahmood, 2001: Phosphorus nutrition of cotton cultivars under deficient and adequate levels in solution culture. Commun. Soil Sci. Plant Anal. 32, 171-187.

Ainsworth, E. A., and A. Rogers, 2007: The response of photosynthesis and stomatal conductance to rising $\left[\mathrm{CO}_{2}\right]$ : mechanisms and environmental interactions. Plant Cell Environ. 30, 258-270.

Almeida, J. F., U. A. Hartwig, M. Frehner, J. Nösberger, and A. Lüscher, 2000: Evidence that P deficiency induces N feedback regulation of symbiotic $\mathrm{N}_{2}$ fixation in white clover (Trifolium repens L.). J. Exp. Bot. 51, 1289-1297.

Barrett, D. J., and R. M. Gifford, 1995: Acclimation of photosynthesis and growth by cotton to elevated $\mathrm{CO}_{2}$ : interactions with severe phosphate deficiency and restricted rooting volume. Aust. J. Plant Physiol. 22, 955-963.

Bélanger, G., A. Claessens, and N. Ziadi, 2011: Relationship between $\mathrm{P}$ and $\mathrm{N}$ concentrations in maize and wheat leaves. Field Crops Res. 123, 28-37.

Bloom, A. J., M. Burger, J. S. R. Asensio, and A. B. Cousins, 2010: Carbon dioxide enrichment inhibits nitrate assimilation in wheat and Arabidopsis. Science 328, 899-903.

Bown, H. E., M. S. Watt, E. G. Mason, P. W. Clinton, and D. Whitehead, 2009: The influence of nitrogen and phosphorus supply and genotype on mesophyll conductance limitations to photosynthesis in Pinus radiata. Tree Physiol. 29, 1143-1151.

Brodribb, T., 1996: Dynamics of changing intercellular $\mathrm{CO}_{2}$ concentration $\left(\mathrm{C}_{\mathrm{i}}\right)$ during drought and determination of minimum functional $\mathrm{C}_{\mathrm{i}}$. Plant Physiol. 111, 179-185.
Campbell, C. D., and R. F. Sage, 2006: Interactions between the effects of atmospheric $\mathrm{CO}_{2}$ content and $\mathrm{P}$ nutrition on photosynthesis in white lupin (Lupinus albus L.). Plant Cell Environ. 29, 844-853.

Cordell, D., J.-O. Drangert, and S. White, 2009: The story of phosphorus: global food security and food for thought. Global Environ. Change 19, 292-305.

FAO, 2010: Food and Agriculture Organization of United Nations (online). http://faostat3.fao.org/home/index. html\#DOWNLOAD. FAO, Rome, Italy (accessed 3 March 2013).

Fleisher, D. H., Q. Wang, D. J. Timlin, J.-A. Chun, and V. R. Reddy, 2012: Response of potato gas exchange and productivity to phosphorus deficiency and carbon dioxide enrichment. Crop Sci. 52, 1803-1815.

Fleisher, D. H., Q. Wang, D. J. Timlin, J.-A. Chun, and V. R. Reddy, 2013: Effect of carbon dioxide and phosphorus supply on potato dry matter allocation and canopy morphology. J. Plant Nutr. 36, 566-586.

Gifford, R., D. Barrett, and J. Lutze, 2000: The effects of elevated $\left[\mathrm{CO}_{2}\right]$ on the C:N and C:P mass ratios of plant tissues. Plant Soil 224, 1-14.

Girma, K., R. K. Teal, K. W. Freeman, R. K. Boman, and W. R. Raun, 2007: Cotton lint yield and quality as affected by applications of N, P, and K fertilizers. J. Cotton Sci. 11, 12-19.

Hewitt, E. J., 1952: Sand and Water Culture. Methods used in the study of plant nutrition. Technical Communication No. 22. Commonwealth Bureau of Horticulture and Plantation, East Malling, Maidstone, Kent. Commonwealth Agricultural Bureaux Farmham Royal, Bucks, UK. pp. 187-190.

IPCC, 2007: Climate Change 2007: the Physical Science Basis. In: S. Solomon, D. Qin, M. Manning, Z. Chen, M. Marquis, K. B. Averyt, M. Tignor, and H. L. Miller, eds. . Contribution of Working Group I to the Fourth Assessment Report of the Intergovernmental Panel on Climate Change. Cambridge University Press, Cambridge, UK and New York, NY, USA.

Jacob, J., and D. W. Lawlor, 1991: Stomatal and mesophyll limitations of photosynthesis in phosphate deficient sunflower, maize and wheat plants. J. Exp. Bot. 42, 1003-1011.

Jin, S. H., J. Q. Huang, X. Q. Li, B. S. Zheng, J. S. Wu, Z. J. Wang, G. H. Liu, and M. Chen, 2011: Effects of potassium supply on limitations of photosynthesis by mesophyll diffusion conductance in Carya cathayensis. Tree Physiol. 31, 1142-1151.

Kawakami, E. M., D. M. Oosterhuis, and J. L. Snider, 2012: Nitrogen assimilation and growth of cotton seedlings under $\mathrm{NaCl}$ salinity and in response to urea application with NBPT and DCD. J. Agron. Crop Sci. 199, 106-117.

Kimball, B. A., and J. R. Mauney, 1993: Response of cotton to varying $\mathrm{CO}_{2}$, irrigation, and nitrogen: yield and growth. Agron. J. 85, 706-712.

Lenka, N. K., and R. Lal, 2012: Soil-related constraints to the carbon dioxide fertilization effect. Crit. Rev. Plant Sci. 31, 342-357. 
Lewis, J. D., K. L. Griffin, R. B. Thomas, and B. R. Strain, 1994: Phosphorus supply affects the photosynthetic capacity of loblolly pine grown in elevated carbon dioxide. Tree Physiol. 14, 1229-1244.

Lichtenthaler, H. K., 1987: Chlorophylls and carotenoids: pigments of photosynthesis. Methods Enzymol. 148, 350-352.

Longstreth, D. J., and P. S. Nobel, 1980: Nutrient influences on leaf photosynthesis: effects of nitrogen, phosphorus, and potassium for Gossypium hirsutum L. Plant Physiol. 65, 541543.

Mullins, G. L., and C. H. Burmester, 1990: Dry matter, nitrogen, phosphorus, and potassium accumulation by four cotton varieties. Agron. J. 82, 729-736.

Prior, S. A., H. A. Torbert, G. B. Runion, G. L. Mullins, H. H. Rogers, and J. R. Mauney, 1998: Effects of carbon dioxide enrichment on cotton nutrient dynamics. J. Plant Nutr. 21, 1407-1426.

Prior, S. A., H. H. Rogers, G. L. Mullins, and G. B. Runion, 2003: The effects of elevated atmospheric $\mathrm{CO}_{2}$ and soil $\mathrm{P}$ placement on cotton root deployment. Plant Soil 255, 179187.

Radin, J. W., and M. P. Eidenbock, 1984: Hydraulic conductance as a factor limiting leaf expansion of phosphorus-deficient cotton plants. Plant Physiol. 75, 372-377.

Radin, J. W., and M. P. Eidenbock, 1986: Carbon accumulation during photosynthesis in leaves of nitrogen- and phosphorusstressed cotton. Plant Physiol. 82, 869-871.

Raghothama, K. G., 1999: Phosphate acquisition. Annu. Rev. Plant Physiol. Plant Mol. Biol. 50, 665-693.

Reddy, K. R., and D. L. Zhao, 2005: Interactive effects of elevated $\mathrm{CO}_{2}$ and potassium deficiency on photosynthesis, growth, and biomass partitioning of cotton. Field Crops Res. 94, 201-213.
Rogers, A., and S. W. Humphries, 2000: A mechanistic evaluation of photosynthetic acclimation at elevated $\mathrm{CO}_{2}$. Global Change Biol. 6, 1005-1011.

Rogers, G. S., L. Payne, P. Milham, and J. Conroy, 1993: Nitrogen and phosphorus requirements of cotton and wheat under changing atmospheric $\mathrm{CO}_{2}$ concentrations. Plant Soil 155-56, 231-234.

Sawan, Z. M., 1986: Effect of nitrogen, phosphorus fertilization and growth regulators on cotton yield and fiber properties. J. Agron. Crop Sci. 156, 237-245.

Saxton, A. M., 1998: A Macro for Converting Mean Separation Output to Letter Groupings in Proc Mixed. 23rd SAS User Group Intl., SAS Institute, NC, USA.

Singh, S. K., and K. R. Reddy, 2011: Regulation of photosynthesis, fluorescence, stomatal conductance and water-use efficiency of cowpea (Vigna unguiculata [L.] Walp.) under drought. J. Photochem. Photobiol. B: Biol. 105, 40-50.

Singh, V., C. K. Pallaghy, and D. Singh, 2006: Phosphorus nutrition and tolerance of cotton to water stress: I. Seed cotton yield and leaf morphology. Field Crops Res. 96, 191-198.

Taub, D. R., and X. Wang, 2008: Why are nitrogen concentrations in plant tissues lower under elevated $\mathrm{CO}_{2}$ ? A critical examination of the hypotheses. J. Integr. Plant Biol. 50, 13651374.

Vance, C. P., C. Uhde-Stone, and D. L. Allan, 2003: Phosphorus acquisition and use: critical adaptations by plants for securing a nonrenewable resource. New Phytol. 157, 423-447.

Zhao, D., D. M. Oosterhuis, and C. W. Bednarz, 2001: Influence of potassium deficiency on photosynthesis, chlorophyll content, and chloroplast ultrastructure of cotton plants. Photosynthetica 39, 103-109. 